\title{
An Improved C-V Model and Application to the Coal Rock Mesocrack Images
}

\author{
Yulong Chen and Hongwei Zhang (iD \\ School of Energy and Mining Engineering, China University of Mining and Technology, Beijing, China \\ Correspondence should be addressed to Hongwei Zhang; hongwei@cumtb.edu.cn
}

Received 30 April 2020; Revised 10 May 2020; Accepted 16 May 2020; Published 17 July 2020

Academic Editor: Zhengyang Song

Copyright (c) 2020 Yulong Chen and Hongwei Zhang. This is an open access article distributed under the Creative Commons Attribution License, which permits unrestricted use, distribution, and reproduction in any medium, provided the original work is properly cited.

\begin{abstract}
In order to accurately and comprehensively obtain information about coal rock mesocrack images, image processing technique based on partial differential equation (PDE) is introduced in order to expound on the active contour model without edges and overcome the deficiency of the $\mathrm{C}-\mathrm{V}$ model. The improved $\mathrm{C}-\mathrm{V}$ model is adopted in order to process mesoimages of coal rocks containing single and multiple cracks and obtain high-quality binary images of coal rock mesocracks and the effective characteristic parameters of coal rock mesostructures through quantitative processing, which will lay solid foundations for the follow-up research into coal rock seepage computation and damage calculation. Studies have shown that, compared to the original $\mathrm{C}-\mathrm{V}$ model, the improved model achieves better image segmentation effects and more accurate quantitative information about coal rock mesostructures for coal rock mesoimages with low contrast ratios and nonuniform grayscale, a fact showing that it can be applied to the calculation of coal rock permeability and damage factors.
\end{abstract}

\section{Introduction}

Underground coal mining will inevitably trigger an inner stress response of coal rock and cause the concentration or release of local stress, thus resulting in the buckling failure of coal rock. In this process, different stress states and amplitudes will bring about different forms of destruction to the rock. The material composition within coal rock and its primary physical and mechanical structure determine its stress and strain status under the external load, which further controls the macromechanical response and failure mechanism. Cracks are generated under high stress in a certain area of underground mining, and the formed cracks of the unstable coal rock in turn affect its inner stress and strain state, thus causing the deflection of local principal stress and improving or worsening the stress state of local coal rock. Research on the damage to coal rock on the mesoscale mainly focuses on the crack initiation, expansion, connection, direction, scale, and properties. These important factors provide significant guidance and design basis for the prevention and control of coal rock instability and failure.
The digital image processing technique provides an effective means for the precise measurement and quantitative analysis of the materials on the mesoscale and opens up a new way for scientists and engineers to gain a comprehensive understanding of the heterogeneity, internal structure characteristics, and morphological characteristics of each component and the corresponding mesomechanic characteristics [1]. The image segmentation algorithms based on threshold segmentation, edge detection, and region growth have been widely applied to the processing of coal rock mesoimage processing. In order to extract the image characteristics of the different compositions of granite, Chen et al. [2,3] proposed a multithreshold region partitioning method based on color space. Yue et al. $[4,5]$ combined digital image processing with numerical methods, such as FEM and FDM, in order to study the influence of the mesostructure of geotechnical engineering materials on inner stress distribution. Zhu et al. [6] also utilized the regional growth algorithm in order to extract the mesostructural information of marbles. Xu et al. [7] used digital image processing technique to study the internal mesostructure characteristics of soil-rock mixture, and by 
using the geometry vectorization transformation technique, the vector concept model that can represent the actual internal mesostructures of soil-rock mixture is constructed. Liu et al. [8] expounded on the rock mesoimage analysis method based on LS-SVM. Yu et al. [9] introduced a digital image processing technique to the analysis system of rock failure processes and adopted a digital image processing technique in order to characterize the heterogeneity of rocks and establish a numerical model reflecting the mesostructure of materials.

Digital image processing includes the removal of noise, contrast enhancement, recovery, segmentation, and characteristic extraction of images through computers. The abovementioned literature review is based on traditional image processing methods, which can inhibit noise but possess the deficiencies of obfuscation of detailed image information, susceptibility to interference, and low accuracy. It is worth noticing that it is inaccurate to process images obtained through coal rock mesomechanic experiments that are characterized by low contrast ratios, multiple details, and weak boundary information, which restricts the fundamental research on the calculation of coal permeability, coal rock damage, and multifield crack coupling. Compared to traditional image processing methods, image processing methods based on partial differential equations (PDE) have the distinctive advantages of conserving the marginal detailed information and realizing nonlinear noise removal while eliminating image noise. PDE-based methods also utilize numerical analysis theories and PDE, which are characterized by high speed, accuracy, and stability. These methods enable analysis from the perspective of the space geometry of the image on the basis of the natural connection between the geometric images and the equation in order to establish an image processing model that is close to reality. The $\mathrm{C}-\mathrm{V}$ model is a widely applied PDE image processing method. This paper introduces and explains the principle of the $\mathrm{C}-\mathrm{V}$ model and proposes an improved model based on image enhancement function. With respect to coal rock mesoimages with nonuniform grayscales and low contrast ratios, the improved $\mathrm{C}-\mathrm{V}$ model obtains highquality segmentation images through processing experiments on mesoimages with single and multiple cracks. This method also obtains coal rock mesostructural information through the binary image quantization process and studies the permeability coefficient and damage variables of coal rocks containing cracks, which provides a reliable support for the in-depth research on the mesomechanic characteristics of coal rocks.

\section{The C-V Model Based on PDE}

2.1. Image Processing Based on PDE. Currently, no universal segmentation theory can be applied to image segmentation technology. The proposed segmentation algorithms are mainly targeted at specific issues. When handing objectives with fuzzy boundaries or dispersed objectives in the image, the traditional segmentation technologies [10] are plagued by low efficiency and precision. If image segmentation is boiled down to an issue related to functional energy minimization, this issue can be converted into a PDE in order to exact solutions through gradient descent flows. The image processing method based on PDE is exercised in accordance with the abovementioned concept. This method can effectively segment the objectives with fuzzy boundaries or dispersed objectives. With the segmentation effect reaching subpixel precision [11], it can automatically detect the inside and outside contours of the image and possess noise immunity. Because of these unique advantages, the image processing method based on PDE has been widely accepted, and it is one of the hot issues in the field of image segmentation. So far, it has been applied to every field of image processing.

The basic concept of the image processing method based on PDE is to evolve an image, a curve, or a curved face in the PDE model and to obtain the expected result by solving this equation [12]. The general type of a PDE of an unknown function $u\left(x_{1}, x_{2}, x_{3}, \cdots, x_{n}\right)$ is as follows [13]:

$$
\sum_{i, j=1}^{n} a_{i j} \frac{\partial u^{2}}{\partial x_{i} \partial x_{j}}+\sum_{i=1}^{n} b_{i} \frac{\partial u}{\partial x_{i}}+c u=f
$$

where $a_{i j}, b_{i}, c$, and $f$ refer to coefficients and $a_{i j}=a_{j i} ; x_{1}, x_{2}$, $x_{3}$, and $x_{n}$ are independent variables and contain a time variable.

2.2. Image Segmentation Based on the C-V Model. The C-V model proposed by Chan and Vese $[14,15]$ has taken into account the certain differences between the average grayscales within the region. If the curve is expressed by a parameter equation, then the segmentation issue can be boiled down to the minimization (the functional of the minimum $\Omega$ area: $\Omega=C+\Omega_{0}+\Omega_{b}$ ) of the energy functional of a closed curve (boundary segmentation):

$$
\begin{aligned}
J\left(C, c_{0}, c_{b}\right) & =\mu \oint_{c} d s+\lambda_{0} \iint_{\Omega_{0}}\left(I-c_{0}\right)^{2} d x d y \\
& +\lambda_{b} \iint_{\Omega_{b}}\left(I-c_{0}\right)^{2} d x d y
\end{aligned}
$$

where $I$ is the image grayscale matrix; $C$ is the closed curve; $\Omega_{0}$ is the internal area of the image divided by $C ; \Omega_{b}$ is the external area; $c_{0}$ and $c_{b}$ refer to the arithmetic mean value of the grayscale of the input image, $I(x, y)$, at $\Omega_{0}$ and $\Omega_{b}$; and $\mu, \lambda_{0}$, and $\lambda_{b}$ are the weight coefficients of each energy item.

Variation level methods are adopted and embedded in function $u$ and introduced in order to obtain the function of the embedded function $u$ :

$$
\begin{aligned}
J\left(u, c_{0}, c_{b}\right) & =\mu \iint_{\Omega} \delta(u)|\nabla u| d x d y \\
& +\lambda_{0} \iint_{\Omega_{0}} H(u)\left(I-c_{0}\right)^{2} d x d y \\
& +\lambda_{b} \iint_{\Omega_{b}}[1-H(u)]\left(I-c_{0}\right)^{2} d x d y
\end{aligned}
$$

where $H(u)$ is the function of Heaviside.

Under fixed function $u$, the following formula is obtained on the basis of the minimum Equation (3) of $c_{0}$ and $c_{b}$ :

$$
c_{i}=\frac{\iint_{\Omega_{i}} I d x d y}{\iint_{\Omega_{i}} d x d y} \quad(i=0, b) .
$$


Under the fixed $c_{0}$ and $c_{b}$, the following formula is obtained under the minimum Equation (3) of $u$ :

$$
\frac{\partial u}{\partial t}=\delta_{\varepsilon}\left[\mu \operatorname{div}\left(\frac{\nabla u}{|\nabla u|}\right)-\lambda_{0}\left(I-c_{0}\right)^{2}+\lambda_{b}\left(I-c_{b}\right)^{2}\right] .
$$

In which,

$$
\delta_{\varepsilon}(u)=\frac{d H_{\varepsilon}(u)}{d u}
$$

where $H_{\varepsilon}(u)$ refers to the arbitrary function that meets the $\lim _{\varepsilon \longrightarrow 0} H_{\varepsilon}(u)=H(u)$. Equations (4) and (5) are synthesized in order to obtain the steady-state solution as well as the segmentation results.

\section{Improved C-V Model}

3.1. Improved $C-V$ Model. The C-V model can effectively detect the weak or fuzzy edge of images, which testifies to the high segmentation accuracy, simple calculation, and noise immunity. These advantages have substantially expanded its applied range. However, the evolutionary velocity $\partial u / \partial t$ of the level set function of the $\mathrm{C}-\mathrm{V}$ model is intimately related to the mean difference $\left(c_{0}-c_{b}\right)$ between the internal and external grayscales of the evolving curve in image $I$. When $c_{0}$ $\approx c_{b}$, or the internal and external grayscales of the evolving curve are close to each other, the early iterations give rise to the slight changes in the level set function $u$; under such circumstances, more iterations are required (sometimes more orders of magnitudes are needed) so as to displace the evolving curve. For the images with nonuniform grayscale and low contrast ratio, such as the coal rock images, the slight grayscale difference between the foreground and the background is prone to cause $c_{0} \approx c_{b}$ in the $\mathrm{C}-\mathrm{V}$ model, thus leading to the low segmentation efficiency. At the same time, the nonuniform grayscale is also prone to cause the segmentation of the background as false foreground, which will greatly reduce the image processing effect. Therefore, for the images, especially the coal rock images with nonuniform grayscale and low contrast ratio, the application of the $\mathrm{C}-\mathrm{V}$ model has been enormously restricted.

In light of the aforesaid analysis, in order to enhance the utilization effect of the $\mathrm{C}-\mathrm{V}$ model, it is necessary to improve the $\mathrm{C}-\mathrm{V}$ model from the two perspectives of increasing the image contrast ratio and the equalization of image grayscale. By mapping the grayscale value, with a narrow grayscale distribution range, of images to be processed into a broadband output value, the contrast ratio of the target area can be effectively improved. The typical methods include logarithm transformation and power transformation. Gonzalez et al. [10] also point out that if the image pixel occupies all the gray levels and are evenly distributed, then the image possesses high contrast and varied gray tone. The image's grayscale is equalized using a histogram equalization method by effectively expanding the common grayscale. When the foreground and background grayscales are close to each other (nonuniform grayscale), the increase in the local con- trast of images (without influencing the overall contrast ratio) will help with the better distribution of image grayscale on the histogram. In the meantime, in the aforesaid operation, the image grayscale value is prone to approach the two ends of the gray level, forming impulse noise. However, noise of this kind must be eliminated in order to maintain the efficiency of level set evolution. The median filtering function adopts the observation window consisting of odd sampling and checks the samples in the input signal in order to judge whether this sample can meet the signal requirements (eliminate the noise). The numerical values in the observation window are subjected to rank ordering with the median in the middle of the observation window as the output. Then, the earliest values are abandoned and new samples are obtained. In this way, the median filtering function can eliminate impulse noise while preserving edge characteristics.

This paper adopts an image enhancement function, $G(I)$, that combines power transformation, logarithm transformation, gray equalization, and a median filtering function to replace the image grayscale matrix, $I$, in the energy function of the original C-V model (Equation (2)). The results are listed below:

$$
\begin{aligned}
J\left(C, c_{0}^{\prime}, c_{b}^{\prime}\right) & =\mu \oint_{c} d s+\lambda_{0} \iint_{\Omega_{0}}\left[G(I)-c_{0}^{\prime}\right]^{2} d x d y \\
& +\lambda_{b} \iint_{\Omega_{b}}\left[G(I)-c_{b}^{\prime}\right]^{2} d x d y
\end{aligned}
$$

where $c_{0}^{\prime}$ and $c_{b}^{\prime}$ represent the grayscale arithmetic mean values of the image at $\Omega_{0}$ and $\Omega_{b}$, respectively. In this way, the image enhancement function, $G(I)$, has increased the difference between the internal and external grayscale averages of the evolving curve $\left(c_{0}^{\prime}-c_{b}^{\prime}\right)$, thus greatly reducing the possibility that the internal and external grayscale averages of the evolving curve are close to each other and obtaining a higher level of set evolutionary velocity and better image processing effects.

The aforesaid image enhancement function, $G(I)$, can be specified as follows:

$$
G(I)=\operatorname{med}\left\{\left(\int_{0}^{F(I)} p_{f}(u) d u\right)_{k} \mid k=1,2,3, \cdots, s \times r\right\},
$$

where $s \times r$ is the size of the median filter, with $s$ and $r$ as odd numbers; $p_{f}(u)$ is the grayscale distribution density function of image $F(I)$; and $G(I)$ is the grayscale matrix of the transformed images of the uniform random variable in $F(I)$ distribution interval, which is monotonic and nondecreasing. $F(I)$ can be expressed as follows:

$$
F(I)=c I^{\gamma}
$$

or

$$
F(I)=c \lg (1+I)
$$

where $c$ is the constant of improving contrast. Equation (9) presents the stretching of contrast based on power 
transformation, and Equation (10) introduces the improved contrast based on logarithm transformation.

By following the $\mathrm{C}-\mathrm{V}$ derivation process, the minimum value of functional $J$ against $u$ is calculated as follows:

$$
\frac{\partial u}{\partial t}=\delta_{\varepsilon}\left[\mu \operatorname{div}\left(\frac{\nabla u}{|\nabla u|}\right)-\lambda_{0}\left[G(I)-c_{0}^{\prime}\right]^{2}+\lambda_{b}\left[G(I)-c_{b}^{\prime}\right]^{2}\right]
$$

In the original $\mathrm{C}-\mathrm{V}$ model, the regularized Heaviside function must be conformed to the condition $\lim _{\varepsilon \rightarrow 0} H_{\mathcal{\varepsilon}}(u)=$ $H(u)$. The improved $\mathrm{C}-\mathrm{V}$ model in this paper selects the following regularized Heaviside function:

$$
H_{\varepsilon}(z)=\frac{1}{2}\left(1+\frac{2}{\pi} \arctan \frac{z}{\varepsilon}\right)
$$

Such regularized function is an odd symmetric function. Parameter $\varepsilon$ is used in order to control the frequency of $H_{\varepsilon}(z)$ and change it from 0 to 1 .

3.2. Numerical Implementation of the Improved $C$ - $V$ Model. The numerical calculation scheme of PDE includes the explicit, implicit, and semi-implicit schemes. The explicit scheme refers to the direct calculation, which can be easily understood but is prone to the accumulation and propagation of error, which testifies to its low algorithm stability. The implicit scheme has the advantage of high stability but usually gives rise to the nonlinear simultaneous algebraic equation. It is usually accompanied by more complicated calculations compared to the explicit scheme. The semiimplicit scheme usually generates a linear difference equation. Different from the nonlinear simultaneous algebraic equation, it usually requires much easier numerical calculation; at the same time, the semi-implicit scheme is also characterized by high stability, and thus, it is widely applied to PDE numerical calculation. Therefore, the semi-implicit scheme is adopted in this paper.

Parameters $\lambda_{0}$ and $\lambda_{b}$ refer to the weight coefficients of each energy item, representing the importance of the segmentation part. The foreground segmentation in image segmentation means the segmentation of the background and vice versa. Therefore, $\lambda_{0}=\lambda_{b}=1$. A discretization scheme combining forward difference and backward difference is adopted with the improved $\mathrm{C}-\mathrm{V}$ model's semi-implicit numerical calculation scheme:

$$
u_{i j}^{t+1}=u_{i j}^{t}+\tau \delta_{\varepsilon} u_{i j}^{t}\left\{\mu P\left(u_{i j}^{t+1}\right)-\left[(G(I))_{i j}-c_{0}^{\prime}\right]^{2}+\left[(G(I))_{i j}-c_{b}^{\prime}\right]^{2}\right\},
$$

$$
\begin{aligned}
P\left(u_{i j}^{t+1}\right) & =D_{x}^{(-)}\left(\frac{D_{x}^{(+)}\left(u_{i j}^{t+1}\right)}{\left[\left(D_{x}^{(+)}\left(u_{i j}^{t}\right)\right)^{2}+\left(D_{y}^{(0)}\left(u_{i j}^{t}\right)\right)^{2}\right]^{1 / 2}}\right) \\
& +D_{y}^{(-)}\left(\frac{D_{y}^{(+)}\left(u_{i j}^{t+1}\right)}{\left[\left(D_{y}^{(+)}\left(u_{i j}^{t}\right)\right)^{2}+\left(D_{x}^{(0)}\left(u_{i j}^{t}\right)\right)^{2}\right]^{1 / 2}}\right) .
\end{aligned}
$$

Under the regularized Heaviside function, the following calculation is applied to the internal and external image grayscale averages of the current evolving curve (zero level set) in order to avoid the troubles of detecting zero level sets:

$$
\begin{aligned}
c^{\prime t} & =\frac{\sum_{i, j} H_{\varepsilon}\left(u_{i j}^{t}\right)(G(I))_{i j}}{\sum_{i, j} H_{\varepsilon}\left(u_{i j}^{t}\right)}, \\
c_{b}^{\prime t} & =\frac{\sum_{i, j}\left[1-H_{\varepsilon}\left(u_{i j}^{t}\right)(G(I))_{i j}\right]}{\sum_{i, j}\left[1-H_{\varepsilon}\left(u_{i j}^{t}\right)\right]},
\end{aligned}
$$

where $c_{0}^{\prime t}$ and $c^{\prime t}{ }_{b}$ represent the image grayscale averages after $t$ iterations.

\section{Experiment Results and Analysis}

4.1. Experiment Process and Results. In order to verify the feasibility and superiority of the method proposed in this paper, both the $\mathrm{C}-\mathrm{V}$ model and the improved $\mathrm{C}-\mathrm{V}$ model were adopted to process images of coal samples with single and multiple cracks with the specific procedure that is listed below:

Step (i) Image Acquisition. The mesoimages of geotechnical materials are usually obtained by the following: (1) digital camera, (2) CCD vidicon with observation made by a stereoscopic microscope or polarizing microscope, and (3) electron microscope (TEM or SEM) [16]. In this study, SEM was used to obtain the mesoimages of coal rock. Figure 1 shows a typical example of a coal containing cracks

Step (ii) Image Segmentation. The C-V model and improved $\mathrm{C}-\mathrm{V}$ model were adopted for the segmentation of the aforesaid images. In this experiment, the semi-implicit scheme was adopted with parameters $\lambda_{0}=\lambda_{b}=1, \varepsilon=1, \mu=250$, and time step $\tau=0.1$. The image segmentation effect based on the $\mathrm{C}-\mathrm{V}$ model is shown in Figure 2 and the image segmentation effect based on the improver $\mathrm{C}-\mathrm{V}$ model in Figure 3. The left images in Figures 2 and 3 introduce the distribution of the evolving curve (zero level set) on the original

in which 

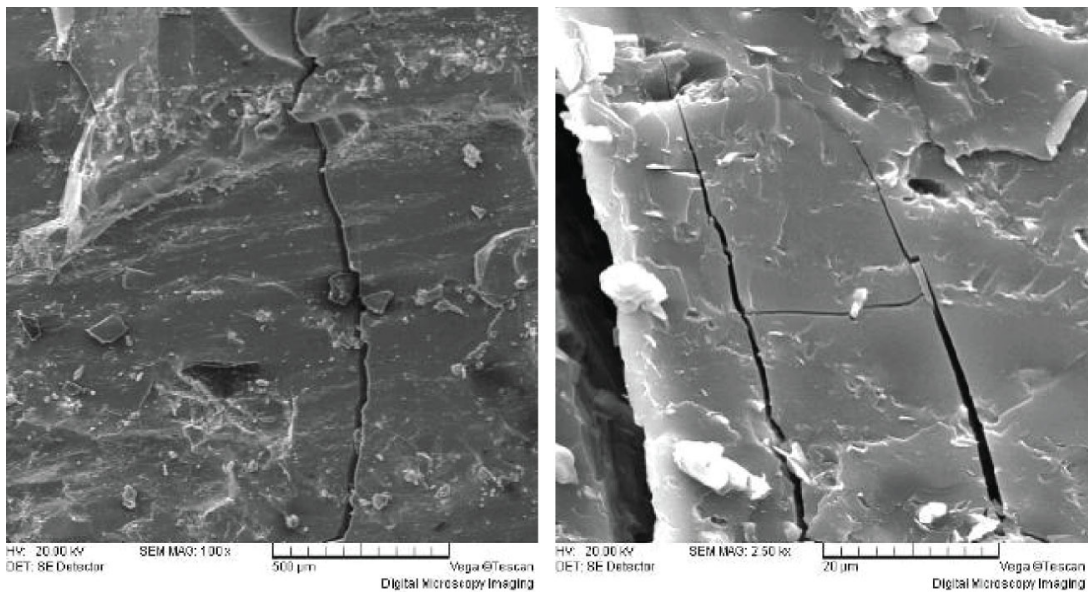

FiguRE 1: Original image of the coal sample containing cracks.
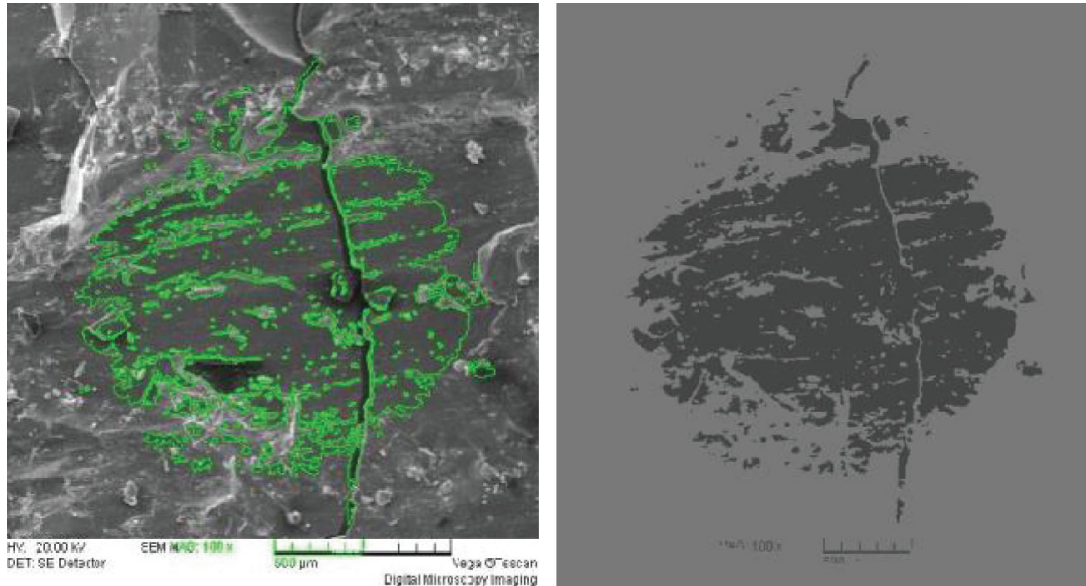

(a) Single crack
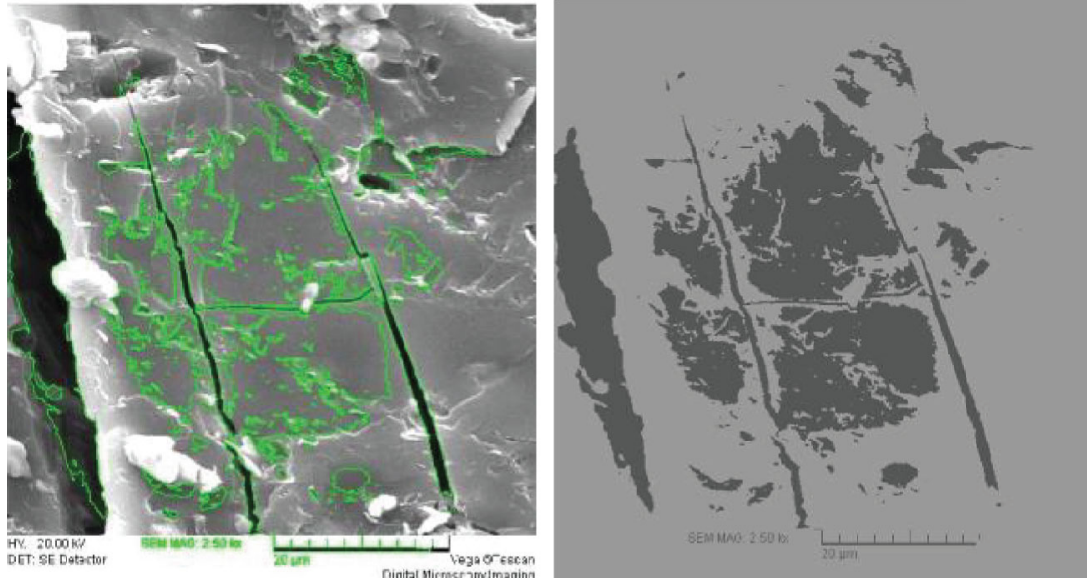

(b) Multicrack

Figure 2: Segmentation of the image by the C-V model (10000 times iteration).

image, and the right images present the image effect of this evolving curve (zero level set).

4.2. Analysis of the Experimental Results. Table 1 presents a comparison of the image segmentation effects and times based on the $\mathrm{C}-\mathrm{V}$ model and the improved $\mathrm{C}-\mathrm{V}$ model.
Table 1 shows that, for both single crack images and multiple crack images, the segmentation based on the $\mathrm{C}-\mathrm{V}$ model does not have an ideal effect while the improved $\mathrm{C}-\mathrm{V}$ model primarily reaches the segmentation effect and obtains a relatively clear crack profile. After 4000 times iterations, the method based on the $\mathrm{C}-\mathrm{V}$ model still fails to reach the ideal 


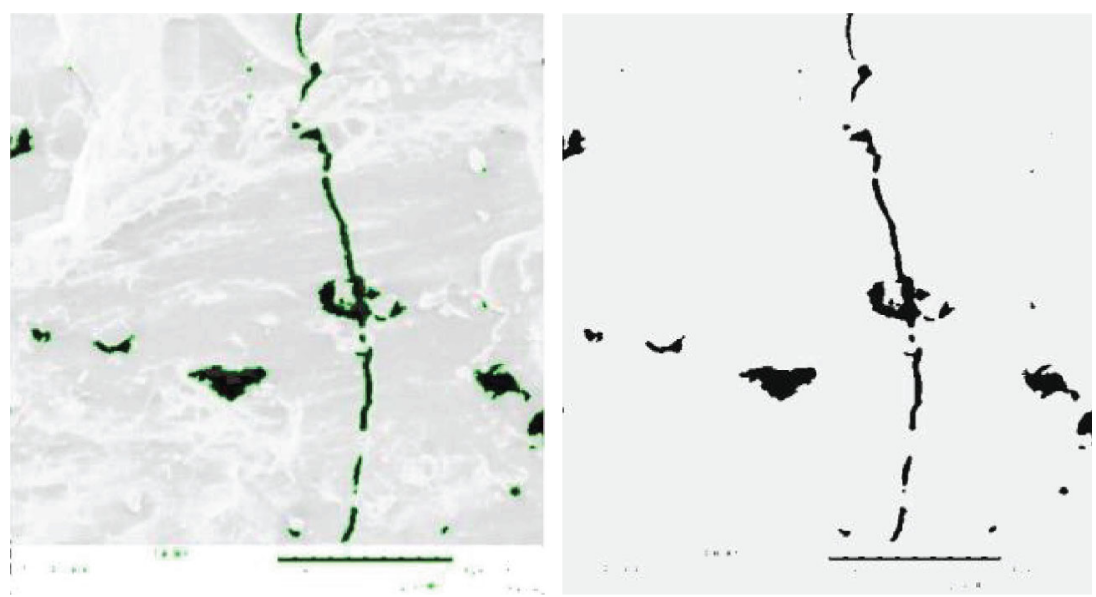

(a) Single crack

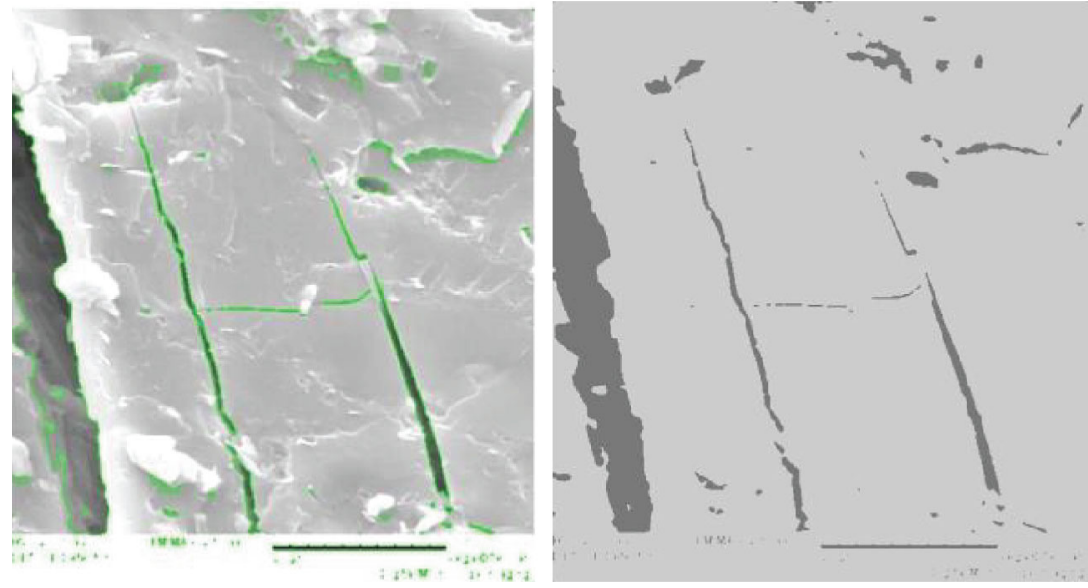

(b) Multicrack

FIgURE 3: Segmentation of the image by the improved C-V model (4000 times iteration).

segmentation effect. Many background details are mixed with the foreground and segmented accompanied by the foreground. At the same time, many details of the foreground are not segmented because they are misunderstood as the background, which results in the failure of crack extraction; however, the improved $\mathrm{C}-\mathrm{V}$ model results in better segmentation effects, as evidenced by the effective extraction of foreground objectives. Despite the fact that only a few background details, such as spots, are captured and distributed, they will be eliminated in the following processing in order to ensure the success of image segmentation. Besides, at the early stages of iteration (about 1000 times), the improved $\mathrm{C}-\mathrm{V}$ model and original $\mathrm{C}-\mathrm{V}$ model require similar amounts of time. Along with the increase in iteration times, especially at 4000 times iterations, the improved $\mathrm{C}-\mathrm{V}$ model consumes 26 seconds less than the original $\mathrm{C}-\mathrm{V}$ method when processing the single crack images and 7 seconds less when processing the multiple crack images. It can be seen that the improved $\mathrm{C}-\mathrm{V}$ model can process the images with nonuniform grayscale and low contrast ratio better than the $\mathrm{C}-\mathrm{V}$ model. Compared to the original $\mathrm{C}-\mathrm{V}$ model, the improved $\mathrm{C}-\mathrm{V}$ model can greatly enhance processing efficiency.

\section{Application of Image Processing Results}

5.1. Crack Information Obtainment. The aforesaid segmentation image based on the improved $\mathrm{C}-\mathrm{V}$ model should be further processed in order to eliminate the interference of the background and obtain more effective crack information. The original image shows that the straight line portion that connects the top and bottom in Figure 3 is the crack and the other black spot-like part is a background detail, not a crack, which should be eliminated by the morphological method.

First, the improved $\mathrm{C}-\mathrm{V}$ model is adopted in order to iterate image binarization for 4000 times and select the segmentation area in accordance with the original image by means of the use of the morphological function, Bwselect. A careful observation shows that the cracks in the original image have been segmented into several sections with the space between each section similar to the line connection. In this paper, the tangential direction growth method is utilized in order to reconnect the fractured cracks, as shown in Figure 4(b). At the same time, the morphological function, Bwmorph, is adopted in order to eliminate the burrs on edges and select the crack profile with the effect shown in 
TABLE 1: Comparison between the effects of the C-V model and the improved C-V model.

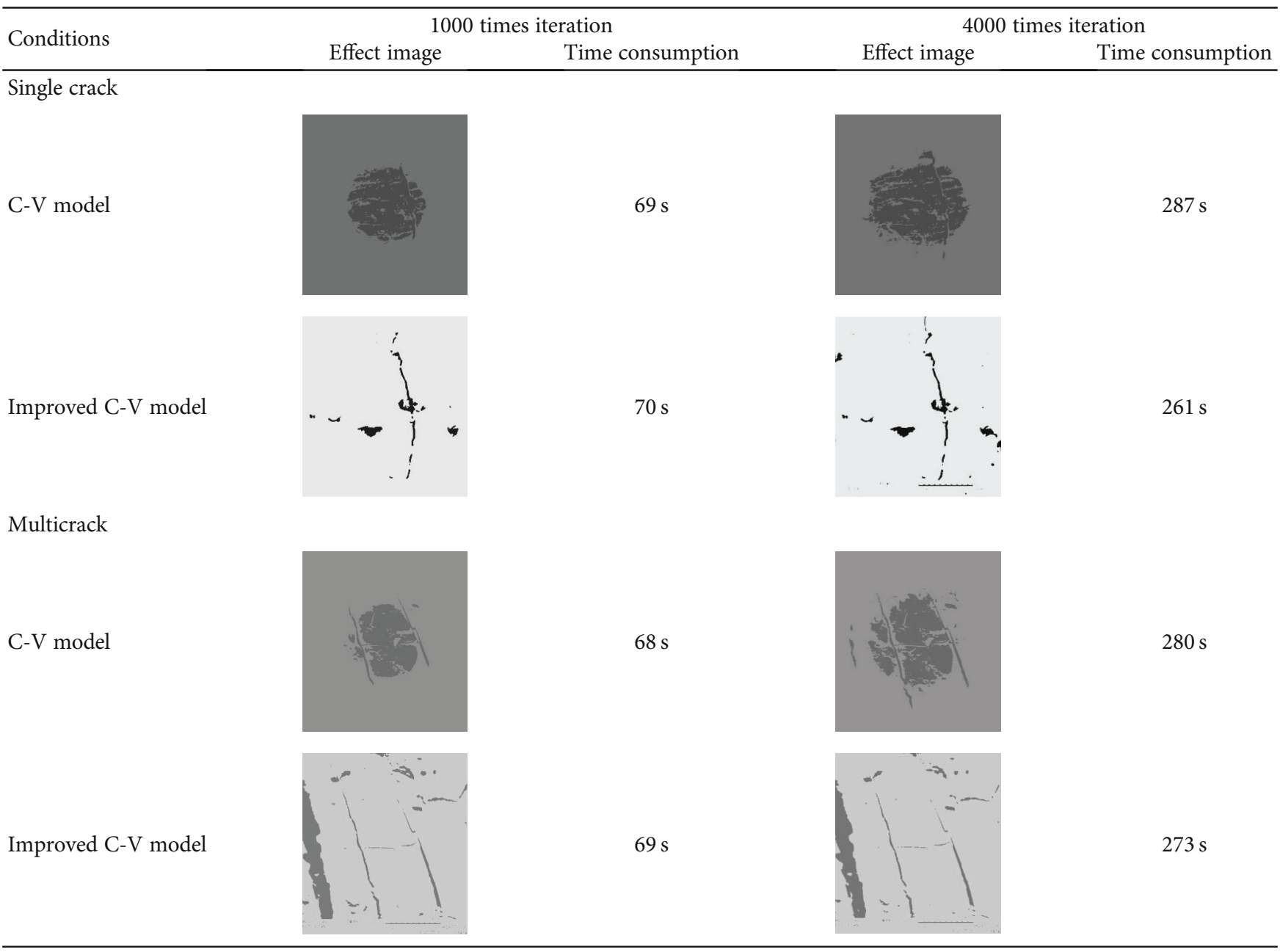

Figure 4(c). Then, the function, Imfill, is used to fill the crack profile with the effect shown in Figure 4(d). At last, Figure $4(\mathrm{e})$ is obtained by overlaying the aforesaid results on the original image. In comparison with the original image, the crack extracted can cover the original crack area, a fact showing the ideal processing results.

The function Regionprops is adopted to obtain the regional characteristic parameters in the image and crack information, as shown in Table 2. According to Table 2, it is convenient to obtain the area of each single-fracture or multiple-fracture crack $(a)$, perimeter $(p)$, long axis length $(l)$, and short axis length $(d)$ with the same standardized second central moment as the area, the radius $(r)$ of the circle with the same area as the area, and the azimuth angle $(\alpha)$. For the multiple crack images, it is also possible to obtain the crack number, $N$, and group them according to the crack strike; it is worth noticing that further processing can obtain the space, $m$, between each group of cracks. Refer to the multiple crack images (Figure 5) for specific operation in this paper with a crack number of $N=4$. In light of the different azimuth angle, these four cracks can be divided into two groups: crack 1, crack 2, and crack 4 in one group and crack 3 in another. Table 2 shows that the average azimuth of cracks 1,2 , and 4 is $-74.4^{\circ}$. Therefore, a straight line is drawn on the binary image along the $15.6^{\circ}$ direction (binary image with the crack) in order to intersect with the aforesaid three cracks. The distance between $A$ and $C$ is measured as 330 pixels; therefore, the space between these groups of cracks is 165 pixels.

\subsection{Further Application of Crack Information Quantification.} The mesocomposition and structure of coal rocks determine their stress-strain states under external force and control their macromechanical response and failure mechanisms. The existence and development of these mesocompositions and structures make the seepage-stress coupling of coal rock very complicated. Digital image processing technology provides an effective method of expressing the heterogeneity of coal rock from the perspective of mesophysical mechanic structure, which has made the mesoresearch on crack rock mass liquid-solid coupling more direct and efficient.

The method based on the improved $\mathrm{C}-\mathrm{V}$ model is adopted in order to process the coal rock image containing cracks and obtain various two-dimensional data about cracks, such as the crack area $(a)$, perimeter $(p)$, azimuth $(\alpha)$, length $(l)$, width $(d)$, number $(N)$, and crack space 


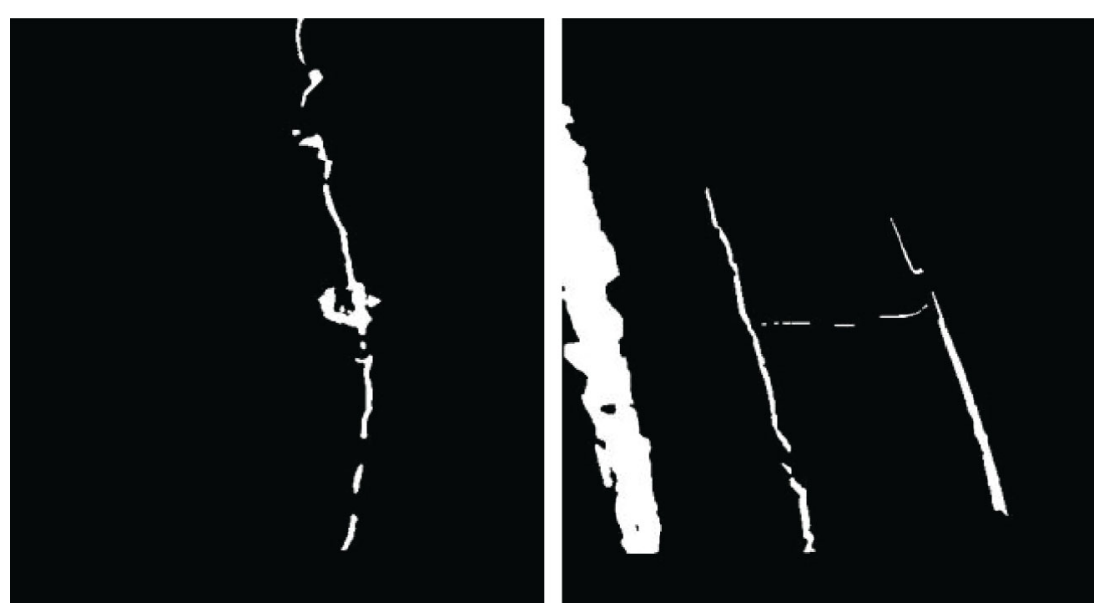

(a) Crack image obtained at the initial stage

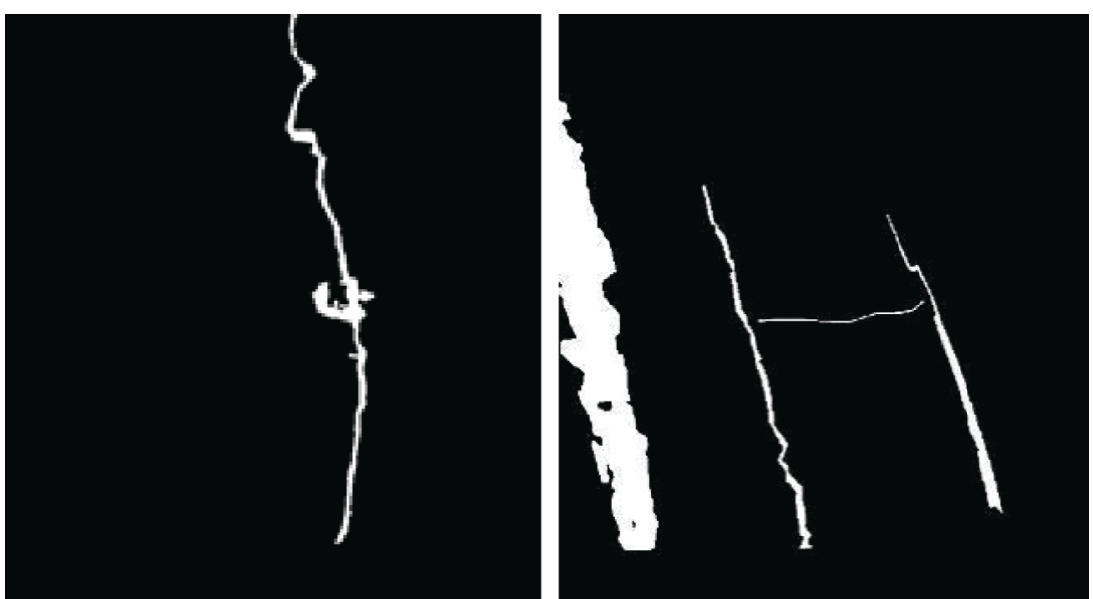

(b) Connection of crack image

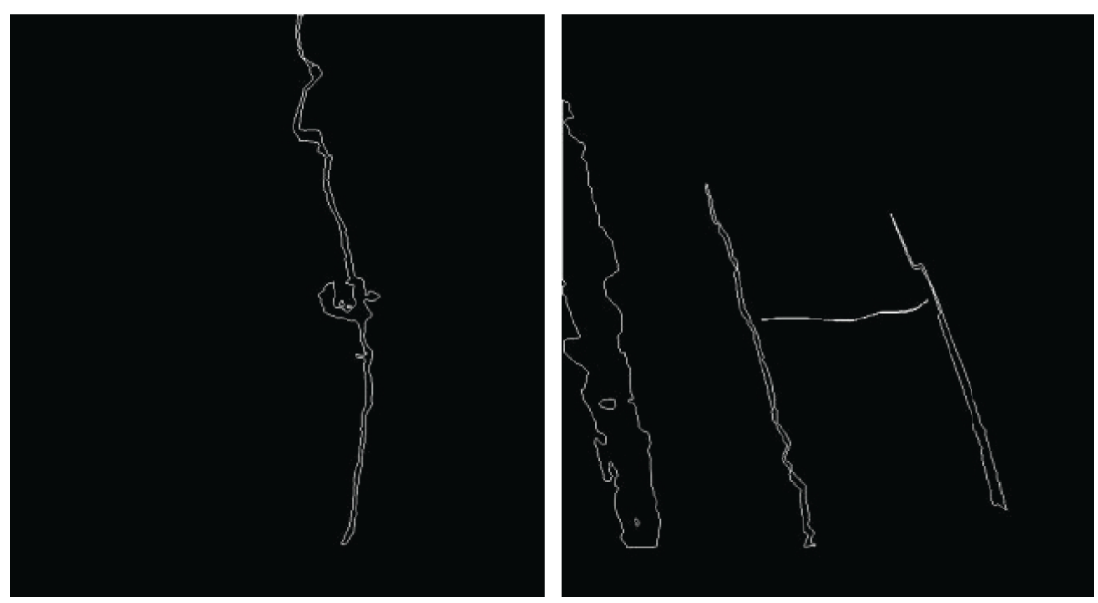

(c) Crack profile

Figure 4: Continued. 


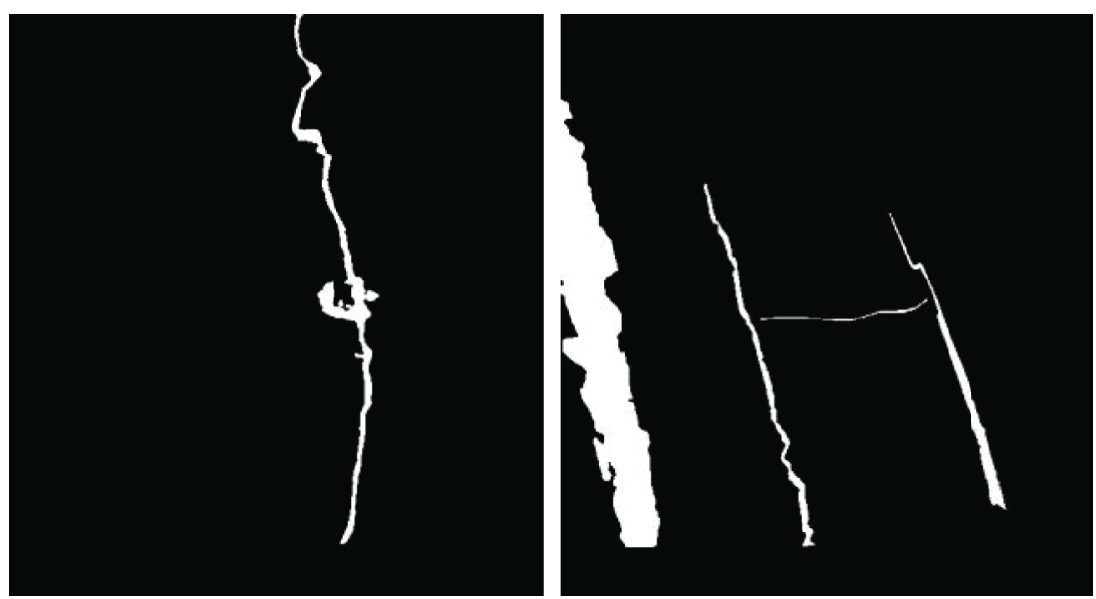

(d) Fill of crack profile
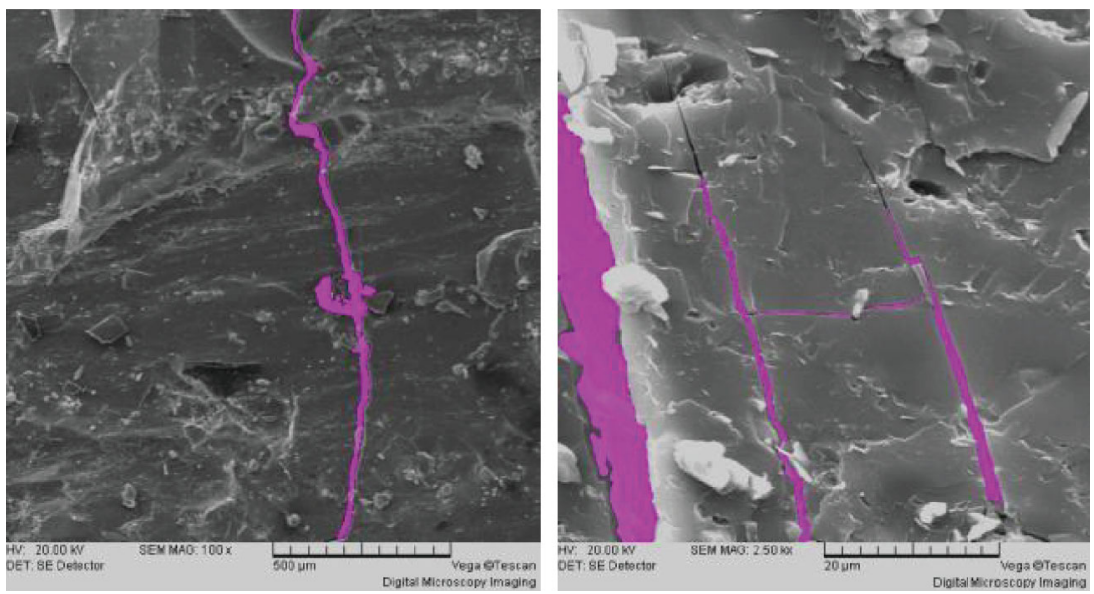

(e) Crack image overlaid on the original one

FIgURE 4: Extraction of crack information.

TABLE 2: Two-dimensional information of the crack (unit: pixel).

\begin{tabular}{lcccc}
\hline Characteristics & Crack 1 & Crack 2 & Crack 3 & Crack 4 \\
\hline Single crack & & & & \\
Crack area & 4175 & & & \\
Fitted long axis & 499 & & & \\
Fitted short axis & 53 & & & \\
Azimuth $\left(^{\circ}\right)$ & -81.9 & & & \\
Crack perimeter & 1385 & & & \\
Equivalent radius & 36.5 & & & \\
Multicrack & & & & \\
Crack area & 16504 & 1888 & 277 & 1942 \\
Fitted long axis & 435 & 418 & 193 & 301 \\
Fitted short axis & 53 & 11 & 11 & 15 \\
Azimuth $\left(^{\circ}\right.$ ) & -79.3 & -73.6 & 4.4 & -70.2 \\
Crack perimeter & 1096 & 816 & 338 & 679 \\
Equivalent radius & 72.5 & 24.5 & 9.5 & 25 \\
\hline
\end{tabular}

$(m)$. An analysis of this information can be used to realize a quantitative description of the mesostructure of coal rock; as a result, this information can also be further

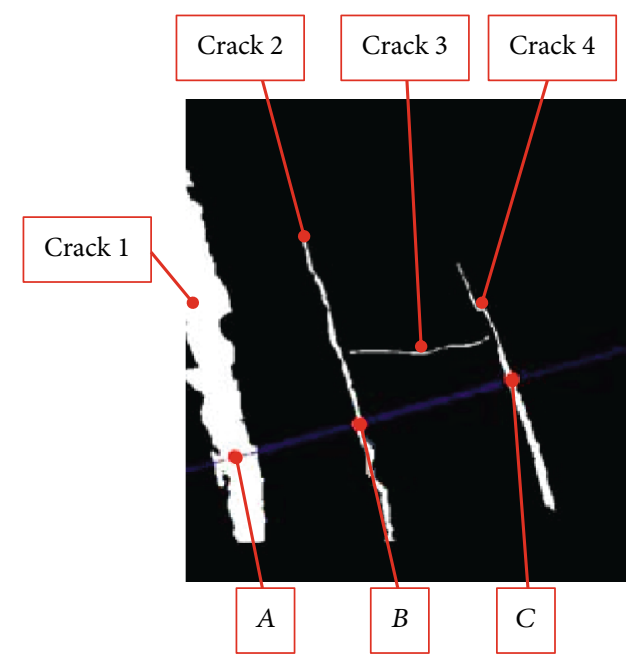

Figure 5: Measurement of the multicrack space.

applied to the theoretical study of the seepage mechanics and damage mechanics of coal rock.

5.2.1. Application of Coal Rock Crack Information to the Research on Seepage Mechanics. In coal rock containing 
cracks, the cracks can be grouped according to their strike. In accordance with the model with equal-width crack, the coefficient of permeability for crack can be expressed as

$$
K_{f}=g d^{2} / 12 v
$$

Romm [17] supposed that the cracked rock mass is a quasicontinuous medium and that the fluid flow conforms to the Darcy law:

$$
q=-K_{f} \frac{d}{m} J^{\prime}
$$

As the Darcy Law suggests,

$$
q=-K_{c} J^{\prime}
$$

Combining Equations (16)-(18) can obtain the coefficient of permeability of the crack group:

$$
K_{e}=\frac{g d^{3}}{12 v m},
$$

where $g$ is the acceleration of gravity, $v$ is the viscosity coefficient of fluid motion, $q$ is the permeation rate, and $J^{\prime}$ is the pressure gradient of fluid.

5.2.2. Application of Coal Rock Information to the Research on Damage Mechanics. The mesostructure of coal rock determines its damage state, which can be roughly obtained by evaluating the mesostructural characteristics of microcracks. The mesoimages obtained through the mesomechanic experiment can be used to quantitatively investigate the relationship among the initiation, expansion, and deformation response of cracks (mesostructure). In order to establish the correlation among the damage variables of mesostructures, macromechanical responses, and physical constitutive equations, variable $\gamma$ should be introduced to express the surface damage degree of a sample and quantify it based on crack data with the expression listed as below:

$$
\gamma(\varepsilon)=\frac{L_{\mathrm{cr}}(\varepsilon)}{L_{\mathrm{cr}}^{f}(\varepsilon)}
$$

where $L_{\mathrm{cr}}^{f}(\varepsilon)$ is the effective length of the crack under the peak stress and $L_{\mathrm{cr}}(\varepsilon)$ refers to the effective length of the crack under the corresponding strain.

The effective length of a crack is calculated according to the following equation [18]:

$$
L_{\mathrm{cr}}^{(1)}=\sum_{i=1}^{n} l_{\mathrm{cr}}^{(i)} \cos ^{2} \beta^{(i)},
$$

where $L_{\mathrm{cr}}^{(1)}$ is the effective length of the surface crack of the sample; $l_{\mathrm{cr}}^{(i)}$ is the length of the $i^{\text {th }}$ crack; $n$ is the total number of cracks; and $\beta^{(i)}$ is the included angle between the $i^{\text {th }}$ crack and the load orientation, i.e., $\beta^{(i)}=\pi / 2-\alpha^{(i)}$, where $\alpha^{(i)}$ is the azimuth of the crack, in the unit of radian. The aforesaid quantitative method of measuring the degree of surface crack damage has effectively simplified data processing without taking into account the anisotropy of coal rock. However, in the practical project, the coal rock is characterized by significant heterogeneity and geometric discontinuity. For this reason, the evaluation of damage of coal rock should also take into consideration the anisotropy. In conformity with the theory of continuum damage mechanics [19-24], the damage tensor, $\tilde{w}$, of the rock mass is defined as follows:

$$
\tilde{w}=\frac{m^{\prime}}{V} \sum_{i=1}^{N} a^{i}\left(n^{i} \times n^{i}\right),
$$

where $m^{\prime}$ is the minimum space of the crack surface, $a$ is the area of the $i^{\text {th }}$ crack, and $n$ is the normal vector of the $i^{\text {th }}$ crack.

\section{Conclusions}

In order to comprehensively and accurately obtain crack information from coal rock mesoimages, this paper introduces image processing technology based on PDE and expounds on the principle of active contour models without edges. In light of the uneven grayscale distribution of the C$\mathrm{V}$ model and the limitations of coal rock images with low contrast ratios, an improved $\mathrm{C}-\mathrm{V}$ model based on image enhancement functions is proposed with a discretization scheme that combines forward difference and backward difference and a semi-implicit scheme for numerical calculation is adopted.

The C-V model and improved $\mathrm{C}-\mathrm{V}$ model were adopted for the image processing of coal rock mesoimages. The results show that the improved $\mathrm{C}-\mathrm{V}$ model achieved a better image segmentation effect and provided more accurate quantitative information about coal rock mesostructure than the $\mathrm{C}-\mathrm{V}$ method for images with both single cracks and multiple cracks. This fact demonstrates that the improved $\mathrm{C}-\mathrm{V}$ model is superior when it comes to processing coal rock mesoimages with nonuniform grayscales and low contrast.

The extracted binary crack images were subjected to quantitative processing in order to extract the length, azimuth, area, perimeter, and space of cracks and crack sets. The quantitative information about crack images at different experimental stages of coal rock mesostructure is used in order to conduct more in-depth research into coal rock seepage and damage mechanics.

\section{Data Availability}

The data used to support the findings of this study are available from the corresponding author upon request.

\section{Conflicts of Interest}

The authors declare no conflict of interest. 


\section{Acknowledgments}

This research was funded by the Beijing Natural Science Foundation (8204068).

\section{References}

[1] C. Chen, X. M. Liu, and C. H. Liu, "Application of digital image processing to rock mesomechanics," Rock and Soil Mechanics, vol. 31, pp. 3468-3472, 2010.

[2] S. Chen, Z. Q. Yue, and L. G. Tham, "Digital image based numerical modeling method for heterogeneous geomaterials," Chinese Journal of Geotechnical Engineering-Chinese Edition, vol. 27, pp. 956-964, 2005.

[3] S. Chen, Z. Q. Yue, and L. G. Tham, "Digital image-based numerical modeling method for prediction of inhomogeneous rock failure," International Journal of Rock Mechanics and Mining Sciences, vol. 41, no. 6, pp. 939-957, 2004.

[4] Z. Q. Yue, S. Chen, and L. G. Tham, "Finite element modeling of geomaterials using digital image processing," Computers and Geotechnics, vol. 30, no. 5, pp. 375-397, 2003.

[5] Z. Q. Yue, S. Chen, H. Zheng, and L. G. Tham, "Digital image proceeding based on finite element method for geomaterials," Chinese Journal of Rock Mechanics and Engineering, vol. 23, pp. 889-897, 2004.

[6] Z. D. Zhu, W. P. Qu, and Z. J. Jiang, "Quantitative test study on mesostructure of rock," Chinese Journal of Rock Mechanics and Engineering, vol. 26, pp. 1313-1325, 2007.

[7] W. J. Xu, Z. Q. Yue, and R. L. Hu, "Study on the mesostructure and mesomechanical characteristics of the soil-rock mixture using digital image processing based finite element method," International Journal of Rock Mechanics and Mining Sciences, vol. 45, no. 5, pp. 749-762, 2008.

[8] Y. B. Liu, S. G. Cao, and Y. C. Liu, "Discussion on analytical method for LS-SVM based mesoscopic rock images," Chinese Journal of Rock Mechanics and Engineering, vol. 27, pp. 1059-1065, 2008.

[9] Q. L. Yu, T. H. Yang, C. Zheng, C. A. Tang, and P. T. Wang, "Numerical analysis of influence of rock mesostructure on its deformation and strength," Rock and Soil Mechanics, vol. 32, pp. 3468-3472, 2011.

[10] R. C. Gonzalez, R. E. Woods, and S. L. Eddins, Digital Image Processing Using MATLAB, Gatesmark Publishing, USA, 2009.

[11] M. Sonka, V. Hlavac, and R. Boyle, Image Processing, Analysis, and Machine Vision, Cengage Learning, USA, 2014.

[12] Q. Q. Ruan and J. Y. Wu, "Partial differential equation(PDE) method on digital image processing," Signal Process, vol. 28, pp. 301-314, 2012.

[13] D. Zhang and G. Chen, Image Processing Based on Partial Differential Equations, Higher Education Press, China, 2004.

[14] T. Chan and L. Vese, "Active contours without edges," IEEE Transactions on Image Processing, vol. 10, no. 2, pp. 266-277, 2001.

[15] T. Chan and L. Vese, "An efficient variational multiphase motion for the Mumford-Shah model," in Proceedings of the 34th Asilomar Conference on Signals, Systems and Computers, pp. 490-494, Pacific Grove, CA, USA, USA, November 2000.

[16] Y. B. Liu, Study on the deformation and damage rules of gasfilled coal base on mesomechanical experiments, Chongqing University, China, 2009.
[17] E. S. Romm, Fluid Flow in Fractures, Nedra Publishing House, Russia, 1966.

[18] P. T. Qi, Z. X. Li, and Y. P. Hang, "Link between compressive deformation and crack propagation in cement mortar," Chinese Journal of Rock Mechanics and Engineering, vol. 22, pp. 425-428, 2003.

[19] J. X. Yuan, "Problems of rock mass damage," Rock and Soil Mechanics, vol. 14, pp. 12-20, 1993.

[20] Z. Y. Song and H. Konietzky, "A particle-based numerical investigation on longwall top coal caving mining," Arabian Journal of Geosciences, vol. 12, no. 18, p. 556, 2019.

[21] Z. Y. Song, H. Konietzky, and M. Herbst, "Three-dimensional particle model based numerical simulation on multi-level compressive cyclic loading of concrete," Construction and Building Materials, vol. 225, pp. 661-677, 2019.

[22] Z. Y. Song, H. Konietzky, and M. Herbst, "Bonded-particle model-based simulation of artificial rock subjected to cyclic loading," Acta Geotechnica, vol. 14, no. 4, pp. 955-971, 2019.

[23] Z. Y. Song, H. Konietzky, and M. Herbst, "Drawing mechanism of fractured top coal in longwall top coal caving," International Journal of Rock Mechanics and Mining Sciences, vol. 130, article 104329, 2020.

[24] Z. Y. Song, W. J. Wei, and J. W. Zhang, "Numerical investigation of effect of particle shape on isolated extracted zone (IEZ) in block caving," Arabian Journal of Geosciences, vol. 11, no. 12, p. 310, 2018. 